Matheus Viezzer Bianchi, Gabriela Fredo, Nelson Junior Tagliari, Ronaldo Viana Leite Filho, Cintia De Lorenzo, Camila Gottlieb Lupion, David Driemeier \& Luciana Sonne

111 Uterine Torsion with a $1080^{\circ}$ Rotation in a Queen with Closed-cervix Pyometra

Sidnei Nunes de Oliveira, Rafaella Miki Hayashi, Felipe Morales Dalanezi, Endrigo Adonis Braga de Araujo, Fabíola Soares Zahn, Luis Fernando Mercês Chaves Silva \& Nereu Carlos Prestes

112 Accumulative Copper Poisoning in Sheep in Northeastern Brazil

Antônio Carlos Lopes Câmara, Robério Gomes Olinda, Luciana Dalcin, Ivana Cristina Nunes Gadelha, Jael Soares Batista \& Benito Soto-Blanco

Luciane dos Reis Mesquita, Leonardo Augusto Lopes Muzzi, Amália Turner Giannico, Ruthnéa Aparecida Lázaro Muzzi, Juliana Fonseca Monteiro \& Paula Desjardins Brienza

114 Post-partum Utero-ovarian Artery Rupture in Mare

Fernanda Maria Pazinato, Bruna da Rosa Curcio, Laura Corrêa Oliveira, Alice Correa Santos, Patrícias Soares Vieira, Sérgio Farias Vargas \& Carlos Eduardo Wayne Nogueira

Bruno Watanabe Minto, Paloma do Espírito Santo Silva, Paulo Vinícius Tertuliano Marinho, Carolina Camargo Zani, Pedro Henrique Ferreira Teles, Karin Werther, Tiago Barbalho Lima \& Júlio Carlos Canola

Aortic Thromboembolism as a Consequence of Bacterial Endocarditis Causing Acute Ischemic Neuromyopathy in a Dog

Gabriel Antonio Covino Diamante, Paulo Vinicius Tertuliano Marinho, Carolina Camargo Zani, Ana Paula Frederico Rodrigues Loureiro Bracarense \& Mônica Vicky Bahr Arias

117 Fibrocartilaginous Embolism Associated with the Extrusion of the Intervertebral Disc in a Dog

Rafael Oliveira Chaves, Bruna Copat, Diego Vilibaldo Beckmann, João Pedro Scussel Feranti, Marília Teresa de Oliveira, Luis Felipe Dutra Corrêa, Angel Ripplinger \& Alexandre Mazzanti

\title{
Squamous Cell Carcinoma in a Pig
}

Maria Viviane Bury dos Santos, Guilherme Konradt, Marilúcia dos Santos Campos, Ariana Lima Pereira, Sóstenes Apolo Correia Marcelino, Pedro Miguel Ocampos Pedroso, Saulo Petinatti Pavarini \& Juliana Targino Silva Almeida e Macêdo

119 Laparoscopic Nephrotomy to Removal of Staghorn Calculus in a Canine

João Pedro Scussel Feranti, Marília Teresa de Oliveira, Luciane Laux Pastore, Hellen Fialho Hartmann, Marcella Teixeira Linhares, Rafael Oliveira Chaves, Luis Felipe Dutra Corrêa \& Maurício Veloso Brun

Aneurysm in the Distal Portion of the Extern Jugular Vein in a Horse

Alice Correa Santos, Fernanda Maria Pazinato, Patricia Soares Vieira, Luciana Oliveira de Araújo, Laura Corrêa de Oliveira, Margarida Buss Raffi, Bruna da Rosa Curcio \& Carlos Eduardo Wayne Nogueira

121 Surgical Correction of Esophageal Stenosis by Right Aortic Arch Persistence in Two Cats

Ítallo Barros de Freitas, Adriana Marks, Felipe Ribeiro Botelho dos Santos, Alceu Gaspar Raiser, Alexandre Mazzanti, Raimy Costa Martins, João Pedro Scussel Feranti \& José Fernando Ibañez

123 Intoxication by Lornoxicam in Two Dogs

Alice Ribeiro Oliveira Lima Albuquerque, Jéssica Fontes Veloso, Ana Paula Fernandes Calazans \& Renata Santiago Alberto Carlos

124 Acute Aflatoxicosis in Swines in Northeastern Brazil

Roberio Gomes Olinda, Joseney Maia Lima, Ricardo Barbosa de Lucena, André Menezes do Vale, Jael Soares Batista, Claudio Severo Lombardo de Barros, Franklin Riet-Correa \& Antônio Flávio Medeiros Dantas 
Olivia Maria Moreira Borges, Valbério Brito Alves, Rosileide dos Santos Carneiro, Sabrina Barros Araujo Dantas, Rodrigo Antônio Torres Matos, Antônio Fernando de Melo Vaz, Armando Marsden Lacerda Filho \& Almir Pereira de Souza

Intermuscular Lipoma in Dogs

Rafael Ricardo Huppes, Natália Dal Pietro, Mônica Carolina Wittmaack, Guilherme Sembenelli, Cynthia Marchiore Bueno, Josiane Morais Pazzini, Paulo César Jark, Andrigo Barboza De Nardi \& Jorge Luiz Costa Castro

Eyelid Apocrine Hidrocystomas in a Cat

João Antonio Tadeu Pigatto, Luciane de Albuquerque, Michelle Petersen Becker, Tanise Carboni da Silva \& David Driemeier

Pentalogy of Fallot in a Crioulo Foal

Ana Carolina Barreto Coelho, Lorena Alvariza Amaral, Carlos Anselmo dos Santos, Jairo Jaramillo Cardenas, Clairton Marcolongo-Pereira, Adriana Lücke Stigger \& Ana Lucia Schild

Mixed Epigastric Axial Pattern Flap Following Cutaneous Hemangiosarcoma Excision in a Male Dog

Jorge Luiz Costa Castro, Vinicius Gonzalez Peres Albernaz, Rafael Ricardo Huppes, Sérgio Santalucia Ramos da Silva, Jair Rodini Engracia Filho \& Andrigo Barboza de Nardi

Femoral Fracture Repair in a Ferret (Mustela putorius furo)

José Arthur de Abreu Camassa, Leonardo Augusto Lopes Muzzi, Fernando Yoiti Kitamura Kawamoto, Camila Cardoso Diogo, Ruthnéa Aparecida Lázaro Muzzi, Raquel de Lima \& Flávia Maria de Oliveira Borges Saad

Right Laparoscopic Adrenalectomy in a Bitch

Fernando Wiecheteck de Souza, Cristiano Gomes, Priscila Natasha Kasper, Marília Teresa de Oliveira, João Pedro ScusselFeranti, Peterson TrichesDornbusch, Rafael Ricardo Huppes, Lucas Lubassinski Daniel, Maurício Veloso Brun, Arícia Gomes Sprada \& Josiane Morais Pazzini

Typhocolitis by Cyathostomins Larvae on a Donkey

Roberio Gomes Olinda, Jefferson da Silva Ferreira, Millena de Oliveira Firmino, Rodrigo Cruz Alves, Antônio Flávio Medeiros Dantas \& Franklin Riet-Correa

Idiopathic Diffuse Lipomatosis in Dogs

Gisele Vieira Sechi, Carolina Zaghi Cavalcante, Marconi Rodrigues de Farias, Jorge Bárcena, Jorge Luiz Costa Castro \& Rebeca Bacchi Villanova

Partial Unilateral Radial Hemimelia in Feline

Hérica Makino, Thalita Priscila Peres Seabra da Cruz, Kalinne Stephanie Bezerra, Samara Rosolem Lima, David Ronald Parra Travagin, Pedro Brandini Nespoli \& Arleana do Bom Parto Ferreira de Almeida

136 Encephalic Meningioma in Dogs

Rafael Oliveira Chaves, Diego Vilibaldo Beckmann, Bruna Copat, João Pedro Scussel Feranti, Marília Teresa de Oliveira, Fernando Wiecheteck de Souza, Marcelo Luís Schwab \& Alexandre Mazzanti

Increased Serum Alkaline Phosphatase in a FeLV-Positive Cat

Stella de Faria Valle, Bárbara Schwarzbach Elesbão, Fernanda Vieira Amorim da Costa, Laís Stefen Gambin, Luciana de Jesus, Mauricio Bianchini Moresco, Marcelle Bettio \& Félix Hilário Diaz González

$138 \quad$ Hepatic Lipidosis in Banded Armadillos (Euphractus sexcinctus) Bred in Captivity

Jael Soares Batista, Carlos Iberê Alves Freitas, Kaliane Alessandra Rodrigues de Paiva, Jardel Bezerra da Silva, Francisco Silvestre Brilhante Bezerra, Roberio Gomes de Olinda, Taciana Melo Fernandes \& Andréia Freitas Oliveira

Ovarian and Uterine Horn Agenesis Accompanied by Ectopic Fetal Mummification in Canine

Thalita Priscila Peres Seabra da Cruz, Samara Rosolem Lima, David Ronald Parra Travagin, Caroline Argenta Pescador \& Roberto Lopes de Souza

140 Canine Rangeliosis: A Rare Case of Hyperparasitemia in the Acute Phase

Danieli Brolo Martins, Aleksandro Schaffer Da Silva, Evelyn de Oliveira, Marcelo Bahia Labruna \& João Fábio Soares

141 Exocrine Pancreatic Carcinoma with Hypoglycemia in a Dog

Gabriela Basilio Roberto, Ane Louise Magro, Meire Christina Seki, Marcos Vinicius Tranquilim \& Liane Ziliotto

142 Spinal T.V.T. Treated with Surgical Excision and Chemotherapy in a Dog

Monica Vicky Bahr Arias, Larissa Garbelini Valentim \& Bianca Ishikawa

Clinical and Pathological Features of Canine Metastatic Adrenocortical Carcinoma

Elisângela Olegário da Silva \& Ana Paula Frederico Rodrigues Loureiro Bracarense 
Fabiana Elias, Rafaela Magalhães Barros, Helvecio Leal Santos-Junior, Rômulo Santos Adjuto Eloi, Vinicius Silva, Fagner Freitas \& Carlos Eduardo Fonseca-Alves

$146 \quad$ Multiple Myeloma in a Dog

Claudine Botelho de Abreu, Rodrigo Bernardes Nogueira, Luiz Eduardo Duarte de Oliveira, Flávia Dada Paiva, Antônio Carlos Cunha Lacreta Junior \& Josilene Nascimento Seixas

147 Osteoblastic Nasal Osteosarcoma in a Wagyu Bovine

Alex dos Santos, Cláudia Cerutti Dazzi, Tanise Policarpo Machado, Eraldo Lourenso Zanella, Ricardo Zanella, Marcio Machado Costa, Rubens Rodriguez \& Adriana Costa da Motta

148 Dioctophyma renale in Dog's Testicle

Bruna Ditzel da Costa Regalin, Ronise Tocheto, Márcia Moleta Colodel, Maria Cecília Camargo, Aldo Gava \& Nilson Oleskovicz

149 Diagnoses of Ovine Infection by the Serotype-4 Bluetongue Virus on Minas Gerais, Brazil

Pamela Aparecida Lima, Kiyoko Uemura Utiumi, Karen Yumi Ribeiro Nakagaki, Daniel Arrais Biihrer, Adriana Silva Albuquerque, Fernanda Rezende Souza, Ana Carolina Diniz Matos, Zélia Inês Portela Lobato, David Driemeier, Ana Paula Peconick, Mary Suzan Varaschin \& Djeison Lutier Raymundo

150 Facial Paralysis Secondary to Hypothyroidism in a Dog

Rafael Oliveira Chaves, Bruna Copat, João Pedro Scussel Feranti, Dênis Antonio Ferrarin, Marcelo Luis Schwab, Luciana Schneider, Raqueli Teresinha França \& Alexandre Mazzanti

\section{Dicephalia in a Bovine}

Marilúcia Campos dos Santos, Zânder Fernandes Teixeira de Azevedo, Ariana Lima Pereira, Maira dos Santos Carneiro Lacerda, Alexandre Redson Soares da Silva, Pedro Miguel Ocampos Pedroso \& Juliana Targino Silva Almeida e Macêdo

Intracranial Transitional Meningioma in a Cat

Nelson Junior Tagliari, Ronaldo Viana Leite Filho, Gabriela Fredo, Luciana Sonne \& Fernanda Vieira Amorim da Costa

Samara Rosolem Lima, Thais Oliveira Morgado, Kalinne Stephanie Bezerra, Fabiana Marques Boabaid, Sandra Hamiro, Leilane Aparecida da Silva, Edson Moleta Colodel \& Nadia Aline Bobbi Antoniassi

\section{Aortic Thromboembolism in a Cat}

Renata Andrade Silva, Tábata Torres Megda, Gabriela Ferreira Siano, Fernanda dos Santos Alves, Suzane Lilian Beier, Bruno Henrique de Albuquerque Paiva \& Ana Patrícia de Carvalho da Silva

Ilium-Femoral Suture Technique Using Wire of Polydioxanone for Hip Joint Stabilization in a Dog with Traumatic Hip Dislocation

Leandro Haczkiewicz Gaiga, João Antonio Tadeu Pigatto, Carla Lempk Constantin \& Talita Susin

156 Cervical Laminectomy for the Treatment of Chronic Caudal Cervical Spondylomyelopathy in a Dog Guilherme Sembenelli, Monica Carolina Nery Wittmaack, Levi Oliveira, Paola Castro Moraes, Bruno Watanabe Minto \& Luis Gustavo Gosuen Gonçalves Dias

157 Equine Sporotrichosis and latrogenic Hypothyroidism

André Giarola Boscarato, Luiz Romulo Alberton, Henrique Augusto Elerbrock dos Santos Komochena, Emerson Botelho, Rita de Cássia Lima Ribeiro Carla Faria Orlandini \& Denis Steiner

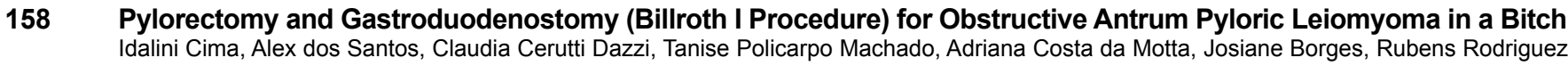
\& Marco Augusto Machado Silva

159 Ureterocolonic Anastomosis in a Dog with Transitional Cell Carcinoma in the Vesical Trigone

Gabriela Friedrich Lobo d'Avila, Elissandra da Silveira, Maria Eduarda Baier, Aline Silva Gouvêa, Natália Fagundes \& Carlos Afonso de Castro Beck 
Detection of Leishmania infantum in Peripheral Blood Smear and Lymph Node of a Domestic Feline

Tamires Ramborger Antunes, Renata Amarilha Valençoela Peixoto, Bruna Brito Oliveira, Simone Sorgatto, Carlos Alberto do Nascimento Ramos \& Alda Izabel de Souza

Anesthetic Management of an Orange-Spined-Hairy-Dwarf-Porcupine (Sphiggurus villosus) Undergoing Myelography

Maria Eduarda Baier, Nelson Junior Tagliari, Bruna Zafalon da Silva, Paula Cristina Sieczkowski Gonzalez, Marcelo Meller Alievi \& Eduardo Raposo Monteiro

Systemic Infection by Pseudomonas aeruginosa in a Dog

Layze Cilmara Alves da Silva, Danielle Aluska do Nascimento Pessoa, Lisanka Ângelo Maia, Rodrigo Antonio Torres Matos \& Meire Maria da Silva Macêdo

YU Pyloroplasty for the Correction of Chronic Hypertrophic Pyloric Gastropathy in Canines

Thalita Priscila Peres Seabra da Cruz, Thaís Ruiz, Wilma Neres da Silva Campos, Ludmila Silva Azevedo, Guilherme de Andrade Bicudo, Pedro Brandini Néspoli \& Roberto Lopes de Souza

Identification of Mycobacterium smegmatis in Bovine Mastitis

Franciele Maboni Siqueira, Cassiane Elisabete Lopes, Gustavo Geraldo Snell \& Marcos José Pereira Gomes

Primary Diffuse Large B Cell Lymphoma in Kidney with Involvement of Central Nervous System and Heart in a Siamese Cat

Priscylla Tatiana Chalfun Guimarães-Okamoto, Maria Claudia Lopes da Silva, Julio Lopes Sequeira, Alessandre Hataka, Fernanda Chicharo Chacar, Silvano Salgueiro Geraldes, José Francisco Antunes Ribeiro \& Alessandra Melchert

Description of Clinical and Surgical Aspects of Four Cases of Lip Commissure to Eyelid Transposition for Repair of Eyelid Coloboma in Cats

Camila do Espirito Santo Maciel, Alexandre Pinto Ribeiro, Thais Ruiz, Deise Cristine Schroder, Thalita Priscila Peres Seabra da Cruz, Nathalie Moro Bassil Dower, Geovanna Barreira Monteiro \& Gabriela Morais Madruga

Reconstructive Surgical Repair of a Forth Degree latrogenic Burn in a Dog

Vinicius Gonzalez Peres Albernaz, Jorge Luiz Costa Castro, Sérgio Santalucia, Rafael Ricardo Huppes, Andrigo Barboza de Nardi \& Josiane Morais Pazzini

Squamous Cell Carcinoma in Third Eyelid of Cat

Maria Eduarda Bastos Andrade Moutinho da Conceição, Danielli Martinelli Martins, Paulo Henrique Leal Bertolo, Daniella Kaísa de Oliveira Bezerra, Carmina Daniela Costa Ferreira da Silva, Lucien Roberta Valente Miranda de Aguirra, Rosemeri de Oliveira Vasconcelos \& Washington Luiz Assunção Pereira

171 Dysphagia Secondary to Spirocerca lupi in a German Shepherd Dog

Carlos Arturo Rodríguez-Alarcón, Andrea López-Aguilar, Federico Pérez-Casio, Ramón Rivera-Barreno, Oliver Castillo-Luna \& Diana Marcela Beristain-Ruiz

Feline Demodicosis by Demodex cati

Marilia Avila Valandro, João Paulo da Exaltação Pascon, Maria Lígia de Arruda Mistieri \& Tiago Gallina

173 Megaesophagus Secondary to Feline Dysautonomia (Key-Gaskell Syndrome) in a Cat

Gabriela da Cruz Schaefer, Daniel Guimarães Gerardi, Neusa Barbosa Castro, Lorena Lima Barbosa Guimarães, Luciana Sonne \& Fernanda Vieira Amorim da Costa

External Skeletal Fixator Intramedullary Pin "Tie-in” for the Repair of Comminuted Tibial Fracture in a Brazilian Fox (Pseudalopex vetulus)

Guilherme Henrique Fernandes Barranco, Michelly Amanda Barssalho, Diego Alaska Almeida, Joana Zafalon Ferreira, Sthephanie Fernandez, Tatiana Morosini de Andrade Cruvinel \& Victor José Vieira Rossetto

Vasectomy in Spotted Paca (Cuniculus paca)

Felipe Farias Pereira da Câmara Barros, Pedro Paulo Maia Teixeira, Maria Eduarda Basto Andrade Moutinho da Conceição, Ricardo Andres Ramirez Uscategui, Leandro Nassar Coutinho, Marina Botelho Soares Brito, Aline Eyko Kawanami \& Wilter Ricardo Russiano Vicente

176 Malignant Metastatic Insulinoma in a Dog

Ozlem Ozmen \& Yusuf Sinan Sirin 
Erick Platiní Ferreira de Souto, Gabrielle Sousa Carvalho, Maria Talita Soares Frade, Roberio Gomes Olinda, Clarice Ricardo de Macêdo Pessoa, Glaucia Denise Kommers, Almir Pereira Souza \& Antônio Flávio Medeiros Dantas

Outbreak of Contagious Echtyma in Sheep in Rio Grande do Sul, Brazil

Welden Panziera, Marina Paula Lorenzett, Márcia Elisa Hammerschmitt, Mônica Slaviero, Henrique Mondardo Cardoso, Saulo Petinatti Pavarini \& David Driemeier

180 Treatment of Two Cats with Advanced Nasal Lymphoma with Orthovoltage Radiation Therapy and Systemic Chemotherapy

Simone Carvalho dos Santos Cunha, Katia Barão Corgozinho \& Ana Maria Reis Ferreira

www.ufrgs.br/actavet

Acta Scientiae Veterinariae. 44: Supplement 1.

2016

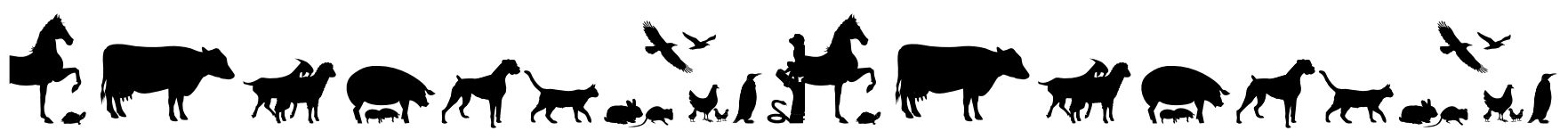

